\title{
STARBURSTS TRIGGERED BY CENTRAL OVERPRESSURE. II. A PARAMETER STUDY
}

\author{
Chanda J. Jog and Mousumi Das \\ Department of Physics, Indian Institute of Science, Bangalore 560 012, India \\ Received 1996 February 15 ; accepted 1996 June 26
}

\begin{abstract}
A parameter study is made of the radiative shock compression of a disk molecular cloud due to the high pressure of the central molecular intercloud medium, after the cloud has fallen into the central region of a galaxy following a galaxy interaction. The dependence of the compression on the disk cloud and central gas parameters has been studied. We show that $f_{\text {shell }}$, the fraction of cloud mass compressed in the outer shell that becomes unstable, is a function of only the external pressure, the cloud radius, and the cloud density. We find that for a wide range of values for the input parameters, $f_{\text {shell }}$ is high and lies between 0.75 to 0.90 . The fraction $f_{\text {shell }}$ is not sensitively dependent on the value of the central gas pressure because the initial inward shock velocity is proportional to the square root of the central pressure, and at later stages it is determined mainly by the self-gravity of the shocked shell. Thus, star formation triggered in disk clouds by compression by the central overpressure, as proposed by Jog \& Das, is a general triggering mechanism and is valid as long as the central gas has an overpressure of even a factor of a few higher than that of the incoming disk molecular clouds.

We apply the mechanism to the galaxies for which the central gas parameters are known; for example, IC 342 and NGC 1808. We find that in both cases a large fraction of the cloud mass will be compressed. Hence, these galaxies should show a central starburst, provided that there is a substantial gas infall rate from the disk to the central region. This agrees with observations, in that NGC 1808 with gas infall due to galaxy interaction has a central starburst, while IC 342 does not.
\end{abstract}

Subject headings: galaxies: interactions — galaxies: ISM - galaxies: starburst — hydrodynamics shock waves — stars: formation - galaxies: ISM

\section{INTRODUCTION}

In an earlier paper (Jog \& Das 1992, hereafter JD92), we proposed a mechanism for the triggering of enhanced massive star formation in the central regions of interacting galaxies. The preencounter gas parameters were taken to be as in the Galaxy. This mechanism was based on the observed fact that the central $1 \mathrm{kpc}$ region in a typical spiral galaxy like the Galaxy contains a diffuse molecular intercloud medium (ICM) (Bally et al. 1988; Stark et al. 1989). JD92 calculate the ICM pressure in the Galaxy and show that it is higher than the effective pressure within a typical giant molecular cloud (GMC) in the disk. Therefore, as a disk GMC falls into the central region following a galactic tidal encounter or due to a bar, it is compressed by the high pressure of the ICM. The compression develops into a radiative shock, and gravitational instabilities develop in the shocked outer layers of the GMC, leading to a burst of star formation. The net resulting luminosity agrees fairly well with the observed values. A similar mechanism for the triggering of star formation by overpressure for the overlapping regions in the case of physically colliding galaxies has been considered by Jog \& Solomon (1992).

JD92 used typical values for the disk GMC and the central ICM parameters in the Galaxy, since the main aim there was to show that a starburst could be triggered in such a case. Recent observations have shown the existence of a diffuse molecular ICM in the central regions of other galaxies, such as, for example, in M33 (Wilson \& Scoville 1990), IC 342 (Downes et al. 1992), and NGC 1808 (Aalto et al. 1994). The ICM thus seems to be a common gas component of spiral galaxies and especially of starburst galaxies. Hence, it is worth extending the earlier study of shock compression (JD92) to cover different disk cloud and ICM parameters, in order to see under which conditions shock compression could occur leading to a starburst. The aim is to see if a starburst triggered by central overpressure is a common phenomenon in galaxies. This is the main motivation for the present work.

We show first that the main physical parameters in the problem are the ICM pressure, the cloud density, and the cloud radius. Since the initial shock velocity is proportional to the square root of the ICM pressure, even for a small overpressure of a factor a few, a shock is driven that then results in star formation in the outer, shocked shell. We find that for a wide range of input parameters, the fraction of cloud mass compressed is high, $\sim 0.75-0.90$. Thus, the triggering of starbursts by overpressure as proposed by JD92 is a general mechanism.

The triggering mechanism is then also applied to the external galaxies IC 342 and NGC 1808. The results for cloud compression from this work indicate that starbursts should be triggered in these galaxies provided there is a high enough gas infall rate from the disk to the central regions.

The range of parameters used for the disk molecular clouds and the central ICM gas are described in $\S 2$. In $\S 3$, the background of the shock compression leading to the onset of gravitational instabilities is given briefly. In $\S 4$, we give first some basic physical results for shock propagation into a cloud. Next we present results for shock compression for the entire parameter range listed in $\S 2$. Then we apply these results to IC 342 and NGC 1808, for which the central gas parameters are known from observations. A few general points are discussed in $\S 5$. The results from this paper are summarized in $\S 6$.

\section{PARAMETERS FOR DISK AND CENTRAL GAS}

In this section, we describe the observed gas parameters of the disk molecular clouds in the Galaxy and the possible 
range of values for the molecular ICM seen in the central regions of spiral galaxies. These are assumed to be representative of preencounter parameters for a typical, undisturbed spiral galaxy.

\subsection{Parameters for Disk Molecular Clouds}

The molecular gas in the disk of the Galaxy is mainly contained in massive GMCs. The parameters describing a GMC are $n_{c}$, the internal number density of hydrogen molecules; $R_{c}$, the cloud radius; and $\sigma_{c}$, the internal, threedimensional velocity dispersion. The sound speed within a cloud is given by $C_{c}=\sigma_{c} / 3^{1 / 2}$.

The number density at the outermost radius of a GMC is taken as $n_{c}=100 \mathrm{~cm}^{-3}$ (Solomon 1978); this is the minimum density for which molecular gas is self-shielded against the galactic UV radiation. Here $\mu_{c}$ is the mean molecular weight in units of amu, $m_{\mathrm{H}}=1.67 \times 10^{-24} \mathrm{~g}$. For a cosmic gas abundance, in which the fraction of helium is $10 \%$ by number of the hydrogen atoms, $\mu_{c}=2.8$ per hydrogen molecule. Hence, $\rho$ the mass density is given as $\rho=$ $m_{\mathrm{H}} \mu_{c} n_{c}$. An average mass density profile $\rho \propto r^{-1}$ that is inversely proportional to the radius is taken for a GMC, as derived by Sanders, Scoville, \& Solomon (1985). Assuming a cloud to be close to virial equilibrium with the above density profile, it can be seen that the internal sound speed is linearly proportional to the cloud radius.

There is a wide range of values observed for $R_{c}$ (Sanders et al. 1985). We take three values for clouds as representing the lower, typical, and upper values for clouds in the Galaxy (Scoville \& Sanders 1987); these give $R_{c}=10 \mathrm{pc}, C_{c}=1.9$ $\mathrm{km} \mathrm{s}^{-1} ; R_{c}=20 \mathrm{pc}, C_{c}=3.8 \mathrm{~km} \mathrm{~s}^{-1}$, which correspond to a "typical" GMC, as defined by Scoville \& Sanders (1987); and $R_{c}=40 \mathrm{pc}, C_{c}=7.6 \mathrm{~km} \mathrm{~s}^{-1}$. The calculated mass of a typical GMC of $20 \mathrm{pc}$ radius is $M_{c}=3.2 \times 10^{5} M_{\odot}$. This agrees with the mass determined observationally from column density measurements (Scoville \& Sanders 1987). A small cloud of $10 \mathrm{pc}$ radius has the mass $M_{c}=4.0 \times 10^{4}$ $M_{\odot}$, and for a large cloud of $40 \mathrm{pc}$ radius it is $M_{c}=2.6$ $\times 10^{6} M_{\odot}$.

The effective gas pressure inside a disk GMC is defined as $P_{\mathrm{GMC}}=m_{\mathrm{H}} \mu_{c} n_{c} C_{c}^{2}$. For a typical GMC, $P_{\mathrm{GMC}}=$ $4.9 \times 10^{5} k_{\mathrm{B}} \mathrm{K} \mathrm{cm}^{-3}$. Note that this is far greater than the thermal pressure within a cloud, and hence this effective pressure offering a higher resistance is considered while calculating the cloud compression by overpressure. The corresponding values of $C_{c}^{2}$ and hence $P_{\mathrm{GMC}}$, for a cloud of $10 \mathrm{pc}$ radius, are smaller by a factor of 4 , and for a cloud of $40 \mathrm{pc}$ radius they are larger by a factor of 4 . Also, as in JD92, we assume that a typical disk GMC reaches the central region with its internal properties undisturbed.

We assume that the parameters of disk GMCs in the Galaxy are representative of GMCs in other spiral galaxies. This is supported by the high-resolution observations of the few individual GMCs that can be resolved in the nearby galaxies such as M31, M33, and IC 10 (Young 1993).

\subsection{Central Gas Parameters}

The molecular gas in the disk radius $\sim 500 \mathrm{pc}$ in the central region of the Galaxy is observed to consist of two distinct components of about equal mass (Bally et al. 1987, 1988). One component consists of massive, high-density clouds with $n_{c} \geq 10^{4} \mathrm{~cm}^{-3}$ (Bally et al. 1988; Güsten 1989). This is $\sim 100$ times larger than the density of the ICM to be discussed next, while the internal random velocity disper- sions of these two components are comparable (Bally et al. 1988). Hence, the ICM pressure is $\sim 100$ times lower than the internal pressure within a central dense cloud. Hence, the ICM cannot compress and trigger star formration in the preexisting dense molecular clouds. Further, due to their low volume filling factor, the high-density central clouds need not be taken into account in considerations of overpressure on the incoming disk GMCs. Thus, the preexisting central dense clouds do not play any role in the triggering mechanism proposed here.

The second component consists of a lower density $\left(n \sim 50 \mathrm{~cm}^{-3}\right.$ ), diffuse, noncloudy molecular gas (Bally et al. 1988; Stark et al. 1989). This diffuse molecular component with a large volume filling factor $(\sim 1)$ has been named the intercloud medium by Stark et al. (1989). JD92 adopted the acronym ICM to denote this component, and for the sake of continuity in notation we shall continue to use it in this paper. Note that the acronym MICM (molecular intercloud medium) would describe the physical properties of this component more accurately.

The origin and energetics of the ICM are not well understood (JD92). The ICM is not self-gravitating. This diffuse gas may originate from the clouds by tidal forces or arise from the massive star formation (JD92), or from diffuse atomic gas compressed into molecular gas, or it may represent the envelopes of nuclear molecular clouds (Aalto et al. 1994).

The average ICM molecular number density, $n_{\mathrm{ICM}}$, is observed to be $\sim 50 \mathrm{~cm}^{-3}$, and the ICM is observed to have a high internal, three-dimensional random velocity dispersion, $\sigma_{\mathrm{ICM}} \sim 30 \mathrm{~km} \mathrm{~s}^{-1}$ (Bally et al. 1988). Hence, the sound speed in the ICM is given by $C_{\mathrm{ICM}}=(1 / 3)^{1 / 2} \sigma_{\mathrm{ICM}}=17.3 \mathrm{~km}$ $\mathrm{s}^{-1}$. The average ICM pressure in the central region of the Galaxy is given by $P_{\mathrm{ICM}}=m_{\mathrm{H}} \mu_{\mathrm{ICM}} n_{\mathrm{ICM}} / C_{\mathrm{ICM}}^{2}=5.1 \times 10^{6} k_{\mathrm{B}}$ $\mathrm{K} \mathrm{cm}^{-3}$. Here $\mu_{\mathrm{ICM}}=2.8$ per hydrogen molecule has been used as discussed above. For the parameter study in $\S 4$, we round off $C_{\text {ICM }}$ for the Galaxy case to $18 \mathrm{~km} \mathrm{~s}^{-1}$; hence, the $P_{\mathrm{ICM}}=5.5 \times 10^{6} k_{\mathrm{B}} \mathrm{K} \mathrm{cm}^{-3}$.

Unlike in the disk GMC case, the values of the average molecular number density in the ICM, $n_{\mathrm{ICM}}$, and the sound speed in the ICM, $C_{\mathrm{ICM}}$, are not coupled so directly, since the ICM is not self-gravitating and is probably not virialized. We consider the range in $n_{\text {ICM }}$ to be $3-100 \mathrm{~cm}^{-3}$. The lower value in this range is the observed value for M33 (Wilson \& Scoville 1990), and $n_{\mathrm{ICM}}=50 \mathrm{~cm}^{-3}$ is the observed value for the central ICM in the Galaxy (Bally et al. 1988). These are the two most nearby galaxies for which high-resolution data are available, so that the $n_{\mathrm{ICM}}$-values are known fairly accurately. Interestingly, this turns out to be roughly the meaningful range for all normal (nonstarburst) spiral galaxies. To sample fully the entire range of ICM pressure, the following values of $n_{\mathrm{ICM}}=3$ $\mathrm{cm}^{-3}, 12 \mathrm{~cm}^{-3}, 50 \mathrm{~cm}^{-3}, 75 \mathrm{~cm}^{-3}$, and $100 \mathrm{~cm}^{-3}$ were chosen. A lower value of, say, $1 \mathrm{~cm}^{-3}$ is not meaningful because this is already the typical value of the average atomic hydrogen number density in the Galaxy (e.g., Spitzer 1978), and hence an ICM of this low a density is not significant. A higher value of $n_{\mathrm{ICM}}$, say, $\geq 100 \mathrm{~cm}^{-3}$, is not taken, since this will lead to an initial shock velocity that is higher than $C_{\mathrm{ICM}}$, and hence the ICM reservoir would no longer remain in pressure contact with the unshocked gas inside the shocked shell in a cloud. This would require a different treatment of the shock propagation. In $\S 4.3$, however, we do apply this mechanism to real galaxies with high observed 
values of $n_{\mathrm{ICM}}$. The validity of this application is discussed in $\S 5$.

The values of the average sound speed in the ICM, $C_{\mathrm{ICM}}$, are taken to lie in the range of $3-18 \mathrm{~km} \mathrm{~s}^{-1}$. The observed values for M33 (Wilson \& Scoville 1990) and the Galaxy (Bally et al. 1988) are given by $\sim 6 \mathrm{~km} \mathrm{~s}^{-1}$ and $\sim 18 \mathrm{~km} \mathrm{~s}^{-1}$, respectively. A value lower than $3 \mathrm{~km} \mathrm{~s}^{-1}$ is not meaningful, since the sound speed in a typical GMC is of this order, and in order that a shock occurs one would need $n_{\mathrm{ICM}}$ to be greater than $n_{c}$. The choice of $3 \mathrm{~km} \mathrm{~s}^{-1}$ as the lower limit for $C_{\mathrm{ICM}}$ is also justified quantitatively because we find in $\S 4.2$ that even this value is not adequate to produce a shock for the range of $n_{\mathrm{ICM}}$-values and the cloud parameters used here. The upper limit of $C_{\mathrm{ICM}}$ is not easy to specify. Physically, the origin and energetics of the ICM are not well understood, as was highlighted in JD92. In starburst galaxies, a value of $C_{\mathrm{ICM}}$ greater than $18 \mathrm{~km} \mathrm{~s}^{-1}$ may be possible, and the physical upper limit may be set by the escape velocity from the bulge. On the other hand, a much higher value than the upper limit used above is probably not meaningful for a disk component, since the rotational speed at $\sim 1 \mathrm{kpc}$ in our Galaxy is $125 \mathrm{~km} \mathrm{~s}^{-1}$ (Sanders 1989). Hence, one would expect $C_{\mathrm{ICM}}$ to be much smaller than the rotational speed. Physically, the two parameters $n_{\mathrm{ICM}}$ and $C_{\mathrm{ICM}}$ feed each other (see JD92), and hence we would expect an ICM of large density to be associated with a large sound speed and vice versa. For the sake of illustrating such cases of high $P_{\mathrm{ICM}}$, we consider a few cases in $\S 4$ in which $C_{\mathrm{ICM}}=30 \mathrm{~km}$ $\mathrm{s}^{-1}$ and $n_{\mathrm{ICM}}=12 \mathrm{~cm}^{-3}$ or $50 \mathrm{~cm}^{-3}$.

\section{GRAVITATIONAL INSTABILITIES IN SHOCKED SHELLS}

When a disk GMC arrives in the central region, it becomes shock compressed by the overpressure of the preexisting central high-pressure gas reservoir. The formulation of the problem is as in JD92. Here the important points from JD92 regarding the compression and the onset of gravitational instabilities in the shocked shell will be summarized.

As shown in JD92, the compression velocity, $V_{s}$, is set by the net momentum transferred per unit area by the difference of pressures in the ICM and inside a disk GMC. It is given by

$$
n_{\mathrm{ICM}} C_{\mathrm{ICM}}^{2}=n_{c} V_{s}^{2},
$$

where $\rho_{\mathrm{R}}=2.8 m_{\mathrm{H}} n_{c}$ is the mass density at the outer edge of a cloud. If $V_{s}>C_{c}$, then the compression develops into a spherical shock. The shock is initially adiabatic, but soon it becomes isothermal for a high enough cooling rate. For an isothermal shock, the shocked gas moves inward with respect to the cloud center with the identical velocity $V_{s}$. Assuming a thin shell approximation for the shocked shell that is valid for high Mach numbers, the equation of motion of the shocked shell of gas is

$$
M_{s} \frac{d V_{s}}{d t}=\frac{G M_{s} M_{\mathrm{un}}}{r^{2}}+\frac{3 G M_{s}^{2}}{r^{2}},
$$

where $r$ represents the radial distance from the cloud center up to which the shock has propagated, $M_{s}$ is the mass of the shocked shell of gas, $V_{s}$ is the shock front velocity, and $M_{\mathrm{un}}$ is the unshocked cloud mass. Note that the second term representing the acceleration due to the self-gravity of the shell becomes important at later stages as more mass is swept into the shell. Assuming a density profile $\rho \propto r^{-1}$ (§ 2.1), we obtain

$$
\begin{gathered}
M_{s}=2 \pi \rho_{R} R_{c}\left(R_{c}^{2}-r^{2}\right), \\
M_{\mathrm{un}}=2 \pi \rho_{R} R_{c} r^{2},
\end{gathered}
$$

where $\rho_{R}$ and $R_{c}$ are the density and radius at the outer edge of an unperturbed cloud. Substituting these in equation (2) and integrating it gives

$$
V_{s}^{2}=A / r+B r-C,
$$

where $A, B$, and $C$ are constants, $A=12 \pi G \rho_{R} R_{c}^{3}$ and $B=$ $8 \pi G \rho_{R} R_{c}$ and $C=20 \pi G \rho_{R} R_{c}^{2}-V_{R}^{2} . V_{R}$ is the shock velocity at the outer edge of the cloud. Note that $V_{R}$ depends only on the square root of $P_{\mathrm{ICM}} / n_{c}$ and does not depend explicitly on the cloud radius. At any later time, $V_{s}$ depends only on $V_{R}, \rho_{R}$, and $R_{c}$. That is, $V_{s}$ is only a function of $P_{\mathrm{ICM}}, \rho_{R}$, and $R_{c}$. As long as $V_{s}<C_{\mathrm{ICM}}$, the ICM pressure reservoir can continue to communicate with the gas inside the cloud.

Integrating equation (5), $t_{c}$, the time taken for the shock to cross to an interior point at a radius $r\left(<R_{c}\right)$ from $R_{c}$, is given by

$$
t_{c}=\int_{R_{c}}^{r} \frac{d r}{\sqrt{A / r+B r-C}} .
$$

We consider next the criterion to be satisfied for the onset of gravitational instabilities in the shocked shell. Assuming a plane-parallel geometry for the shell, a local linear perturbation in the plane of the shell obeys a dispersion relation similar to that obtained for the stability of thin galactic disks (Toomre 1964; Jog \& Solomon 1984):

$$
\omega^{2}=k^{2} C_{c}^{2}-2 \pi G k \Sigma_{0},
$$

where $k$ and $\omega$ are respectively the wavenumber and the angular frequency of the perturbation. The surface density of the shell, $\Sigma_{0}$, is given by the mass of the shell (eq. [3]) divided by its surface area $\left(4 \pi r^{2}\right)$ to be

$$
\Sigma_{0}=\frac{\rho_{R} R_{c}}{2}\left(\frac{R_{c}^{2}}{r^{2}}-1\right) .
$$

The perturbation is unstable when $\omega^{2}<0$. The wavenumber, $k_{\max }$, for the most unstable mode is obtained by solving $d \omega^{2} / d k=0$ and is given by

$$
k_{\max }=\pi G \Sigma_{0} / C_{c}^{2} .
$$

Substituting $k_{\max }$ in the dispersion relation (eq. [7]), we obtain the time of growth or the $e$-folding time for the most unstable mode, $t_{g}$, to be

$$
t_{g}=\frac{C_{c}}{\pi \Sigma_{0} G} .
$$

As the shocked shell propagates inward, $t_{c}$, the crossing time, increases. On the other hand, $t_{g}$, the growth time for instabilities, is inversely proportional to the surface density of the shell, and hence as the shell moves inward accreting more cloud mass as it goes along, $t_{g}$ decreases. At the transition or crossover point of the curves for $t_{c}$ and $t_{g}$ versus decreasing radius, that is, when $t_{c}=t_{g}$, the shocked shell becomes gravitationally unstable and begins to fragment. Further nonlinear evolution of this fragmenting, highdensity shell can result in star formation (JD92).

Define $f_{\text {shell }}$ to be the fraction of the cloud mass compressed into the shocked shell at the transition or crossover 
point of these two curves:

$$
f_{\text {shell }}=M_{s} / M_{c} \text {. }
$$

Thus, $f_{\text {shell }}$ defines the fraction of cloud mass that gives rise to the starburst event in a cloud. The total mass of stars formed depends on the star formation efficiency (SFE) in the dense, shocked shell (see JD92). The net luminosity from the central region depends on the rate of gas infall from the disk region to the central region (JD92).

A necessary condition for gravitational instabilities to grow in the shocked shell of gas is that $\lambda$, the most unstable wavelength $\left(=2 \pi / k_{\max }\right.$, where $k_{\max }$ is defined by eq. [9]), be less than $l_{\text {shell }}=2 \pi r$, the perimeter of the unshocked gas at the crossover point of the $t_{c}$ and $t_{g}$ curves. Unless this condition is satisfied, the assumption of a two-dimensional geometry, and hence the two-dimensional dispersion relation (eq. [7]) used to study the onset of instabilities is not valid. This condition was assumed implicitly to be satisfied in JD92. We show in $\S 4$ that this condition is satisfied for the parameter range considered here.

\section{RESULTS}

\subsection{Basic Features of Cloud Compression}

\subsubsection{Input Parameters}

The shock velocity $V_{s}$, and hence the crossing time $t_{c}$ to reach a certain radius $r$ inside a cloud, depend on only three input parameters: $P_{\mathrm{ICM}}$, the ICM pressure, $\rho_{R}$, the cloud density at the outer edge, and $R_{c}$, the radius of the cloud, as was shown in $\S 3$. For a virialized cloud with a density profile $\rho$ proportional to $1 / r$, the internal sound speed $C_{c}$ is linearly proportional to $R_{c}$, the radius of the cloud $(\S 2.1)$. Hence, $t_{g}$, the time taken to reach a given radius, depends only on the density profile in the cloud, i.e., on $\rho_{R}$, and $R_{c}$, as can be seen from equation (10). Therefore, the crossover point, at which $t_{c}=t_{g}$, is a function only of $P_{\mathrm{ICM}}, \rho_{R}$, and $R_{c}$. Thus, these three are the main input parameters in the problem. Hence, it is easy to span the entire parameter space to cover values for these parameters; see $\S 4.2$.

Note also that for virialized clouds with a $1 / r$ density profile, the effective internal pressure in a cloud, $P_{\mathrm{GMC}}$ (see $\S 2.1$ ), depends only on $\rho_{R}$ and $R_{c}$. Hence, the quantity $P_{\mathrm{ICM}} / \boldsymbol{P}_{\mathrm{GMC}}$, or the overpressure on a cloud, is also an independent input parameter.

\subsubsection{Shock Propagation}

The behavior of the various resulting parameters as the shock is driven into a cloud is discussed next. In Figure 1, we plot $V_{s}$, the shock velocity, versus $\left(1-r / R_{c}\right)$, the fractional shock penetration depth within a cloud (i.e., the fractional radius measured from the outer edge of the cloud), for a typical disk cloud in our Galaxy that has fallen into the central $1 \mathrm{kpc}$ region, so that $n_{c}=100 \mathrm{~cm}^{-3}, R_{c}=20 \mathrm{pc}$, and $P_{\mathrm{ICM}}=5.5 \times 10^{6} k_{\mathrm{B}} \mathrm{K} \mathrm{\textrm {cm } ^ { - 3 }}$. The corresponding plots of $t_{c}$ and $t_{g}$ versus penetration depth, and the most unstable wavelength over the perimeter of the unshocked cloud, namely, $\lambda / 2 \pi r$ versus penetration depth, are given in Figures 2 and 3 , respectively.

Recall that the initial shock velocity $V_{R}$ set by the ICM pressure is proportional to the square root of $P_{\mathrm{ICM}} / n_{c}(\S 3)$. Initially as the shock is driven in, the shock velocity increases slowly due to the gravitational acceleration due to the unshocked cloud (the first term on the right-hand side of eq. [2]). Later on, when a substantial mass has been driven

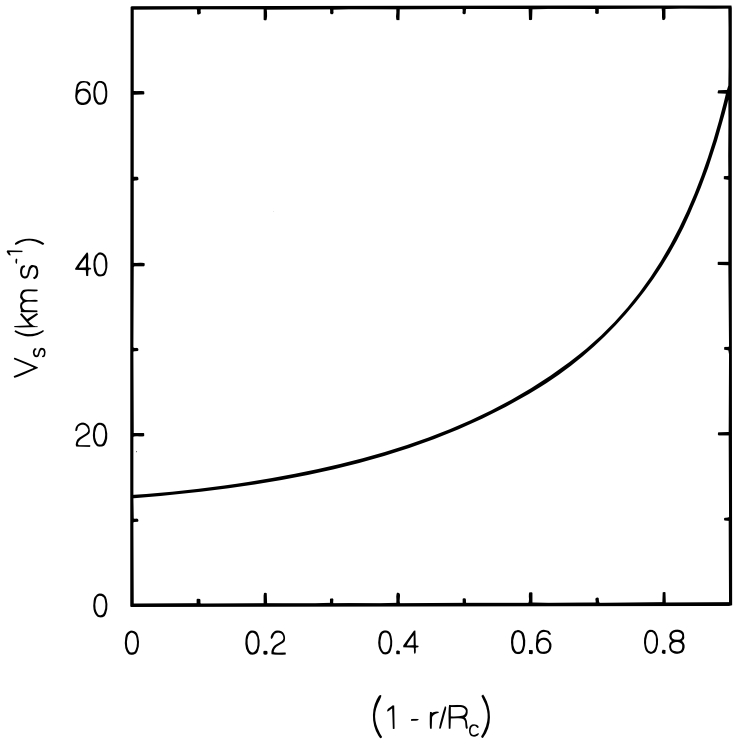

FIG. 1.-A plot of $V_{s}$, the shock velocity, vs. $\left(1-r / R_{c}\right)$, the fractional cloud penetration depth of the shock measured from the outer edge of the cloud, for a typical disk cloud that has fallen into the central region of the Galaxy, so that $P_{\mathrm{ICM}}=5.5 \times 10^{6} k_{\mathrm{B}} \mathrm{K} \mathrm{cm}{ }^{-3}$ and $R_{c}=20 \mathrm{pc}$. Once the shocked shell contains substantial mass, the self-gravity of the shell becomes important in the determination of $V_{s}$, and the shock velocity rises rapidly.

into the postshock shell, the self-gravity of the shell (the second term on the right-hand side of eq. [2]) is the dominant force driving the shock inward. Hence, $V_{s}$ increases rapidly, indicating a rapid acceleration of the shocked shell (see Fig. 1).

Hence, the curve for the shock crossing time, $t_{c}$, versus the shock penetration depth rises slowly initially as the shock is first driven in by the shock velocity set by the

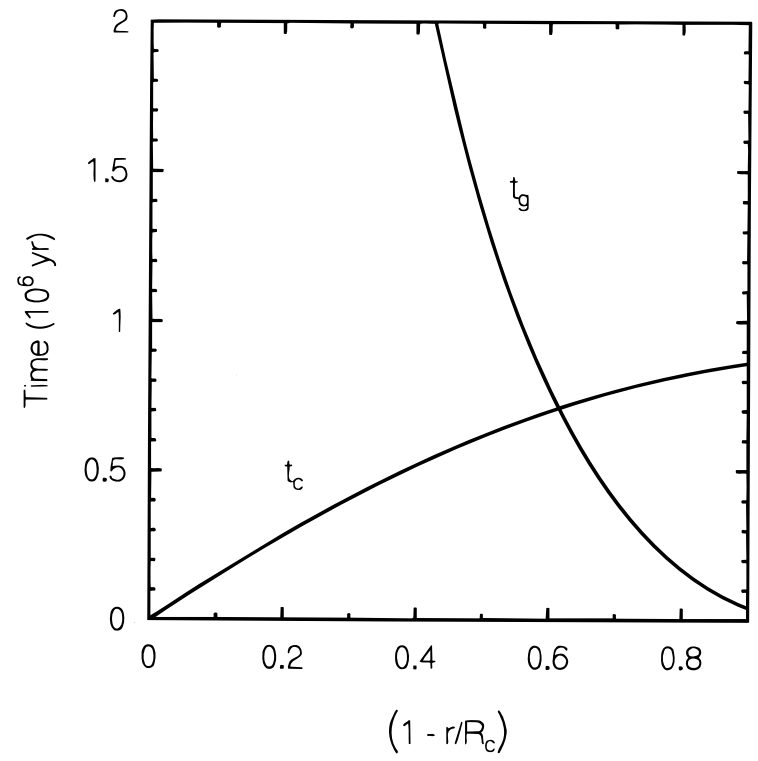

Fig. 2.-A plot of $t_{c}$, the crossing time, and $t_{g}$, the growth time, of the fastest growing instability in the shocked shell, vs. $\left(1-r / R_{c}\right)$, the fractiona cloud penetration depth for the parameters as in Fig. 1. Here $t_{c}$ increases slowly at first and saturates later on, when the shock velocity shoots up (see Fig. 1); $t_{q}$ decreases sharply at first, since the rate of increase of the shocked shell surface density is high, and it drops more gradually as the shock is driven in further. The instabilities set in at the crossover point at which $t_{c}=t_{g}$; here $f_{\text {shell }}=0.85$. 


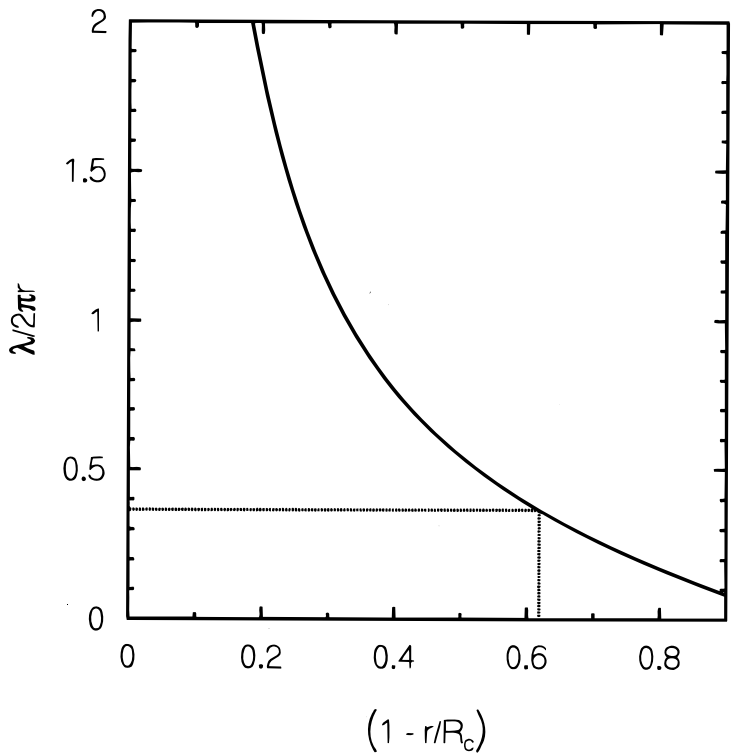

FIG. 3.-A plot of $\lambda / 2 \pi r$ vs. $\left(1-r / R_{c}\right)$, the fractional cloud penetration depth, where $\lambda$ is the size of the fastest growing instability in the shocked shell and $2 \pi r$ is the perimeter of the unshocked cloud, for the parameters as in Figs. 1 and 2. This ratio is $0.37(<1)$ at the crossover point as shown by the dotted line; thus, the use of the two-dimensional dispersion relation to study the onset of gravitational instabilities is justified.

external ICM pressure. As the self-gravity term becomes important in the determination of shock velocity, the shock moves in quickly, and hence $t_{c}$ saturates to a slowly increasing function of the depth (see Fig. 2).

The variation in $t_{g}$ with depth depends only on the internal cloud density profile (see $\S$ 4.1.1). Here $t_{g}$ is inversely proportional to $\Sigma_{0}$, the surface density of the shell. $\Sigma_{0}$ is small to begin with; hence, as the shock is driven in, even a small initial increase in $\Sigma_{0}$ causes a sharp decrease in $t_{g}$. This can be confirmed from the fact that the magnitude of $d t_{g} / d r$ is proportional to $r /\left(R_{c}^{2}-r^{2}\right)^{2}$ and hence is large near the cloud edge. As the shock is driven in further, accreting more cloud mass as it goes along, $\Sigma_{0}$ increases substantially, and hence $t_{g}$ drops more gradually with decreasing radius. The crossover between the two curves in Figure 2 occurs at $r / R_{c}=0.38$; at this point, the fractional cloud mass in the shocked shell, $f_{\text {shell }}$, is 0.85 .

From Figure 3 it can be seen that at the crossover point (indicated by the dashed line), the ratio $\lambda / 2 \pi r$ is 0.37 , which is $<1$; hence, the two-dimensional dispersion relation (eq. [7]) used to study the onset of gravitational instabilities is valid (see $\S 3$ ). This is also true for the parameter range we consider in $\S 4.2$. For clouds with a higher compression so that $\Sigma_{0}$ is higher, and for smaller clouds so that $C_{c}$ is lower, the above condition is satisfied even better.

The above basic physical features of the shock propagation will be used to explain the results obtained in $\S 4.2$.

\subsection{Cloud Compression: Parameter Study}

Here we obtain the results for cloud compression by overpressure for the entire parameter space. Recall from § 4.1.1 that the main input parameters in the problem are $P_{\text {ICM }}$, the external ICM pressure; $\rho_{R}$, the cloud number density at the outer edge of a cloud; and $R_{c}$, the cloud radius. We keep $\rho_{R}$ constant as per the discussion in $\S 2.1$ and study the variation of the resulting shock compression with $P_{\mathrm{ICM}}$ and $R_{c}$, the cloud radius. $P_{\mathrm{ICM}}$ is varied by chang- ing the ICM number density, $n_{\mathrm{ICM}}$, and the ICM sound velocity, $C_{\mathrm{ICM}}$, within the limits discussed in $\S 2.2$. Recall from $\S 3$, equation (11), that $f_{\text {shell }}$ is defined to be the fraction of cloud mass compressed in the outer shell at the point of onset of the shell instability. In Figure 4 we plot results for $f_{\text {shell }}$ versus $P_{\text {ICM }}$ for clouds of radii $R_{c}=10 \mathrm{pc}, R_{c}=20 \mathrm{pc}$, and $R_{c}=40 \mathrm{pc}$. This figure illustrates how clouds of different masses respond to the pressure of the ICM.

\subsubsection{Dependence on $P_{\mathrm{ICM}}$}

Figure 4 shows that for a cloud of given radius, and hence a given $P_{\mathrm{GMC}}, f_{\text {shell }}$ increases at first as $P_{\mathrm{ICM}}$ is increased. This is because the initial shock velocity is set by $P_{\mathrm{ICM}}$ to be proportional to $P_{\mathrm{ICM}}^{1 / 2}$; see equation (1), $\S 3$. Therefore, the crossing time $t_{c}$ up to a given radius is smaller. The curve for $t_{g}$, on the other hand, just depends on the cloud internal density profile and hence is independent of $P_{\mathrm{ICM}}$. Therefore, the crossover point of the $t_{c}$ and $t_{g}$ curves occurs deeper into a cloud. Thus, the shock is driven deeper into a cloud leading to a higher $f_{\text {shell }}$ as $P_{\text {ICM }}$ is increased.

Now once the shocked shell contains a substantial amount of mass, the self-gravity of the shell is the dominant effect in setting the shock velocity and hence in setting the subsequent values of $t_{c}$. Thus, for large values of $P_{\text {ICM, }}$, during most of the compression, the shock velocity and the compression time $t_{c}$ is set by the self-gravity of the shell. Hence, $t_{c}$ and the actual crossover point become nearly independent of the initial shock velocity or the value of the $P_{\text {ICM }}$. This is why the resulting $f_{\text {shell }}$ saturates at large values of the ICM pressure.

\subsubsection{Dependence on Cloud Mass}

Figure 4 shows that at a given $P_{\mathrm{ICM}}, f_{\text {shell }}$ is higher for a smaller mass cloud. Recall that for a virialized cloud of $1 / r$ density profile, the internal sound speed is proportional to the cloud radius $(\$ 2.1)$. Hence, $t_{g}$, the time for growth of instabilities at a given $r$, is proportional to $r^{2} /\left(R_{c}^{2}-r^{2}\right)$; see equation (10). Hence, the $t_{g}$-value is higher at a given radius

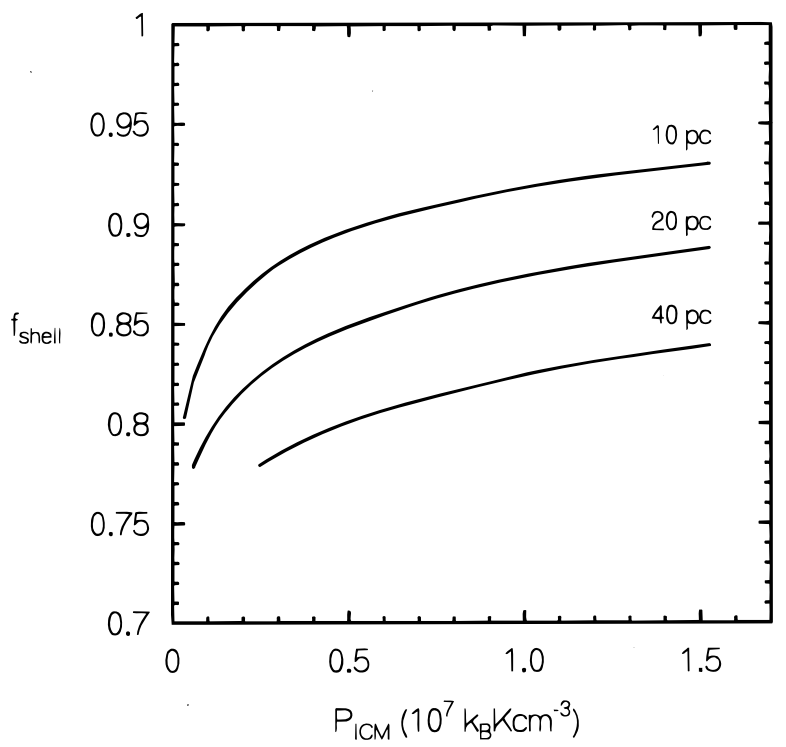

FIG. 4.-A plot of $f_{\text {shell }}$, the fraction of cloud mass compressed at the point of onset of shell instabilities, as a function of the ICM pressure, $P_{\text {ICM }}$, for clouds of radii $10 \mathrm{pc}, 20 \mathrm{pc}$, and $40 \mathrm{pc}$. Here $f_{\text {shell }}$ increases slowly with the increasing $P_{\mathrm{ICM}}$ at first and saturates for higher $P_{\mathrm{ICM}}$ values. For a smaller mass cloud, $f_{\text {shell }}$ is higher, and the saturation in $f_{\text {shell }}$ sets in at a lower value of $P_{\mathrm{ICM}}$. 
in the case of a smaller cloud. Therefore, the crossover between the two curves occurs deeper for the smaller mass cloud. Hence, for the same value of $P_{\text {ICM }}$, the resulting $f_{\text {shell }}$ is higher for a smaller mass cloud.

Figure 4 also shows that for a smaller mass cloud, the saturation in $f_{\text {shell }}$ occurs at a smaller value of $P_{\mathrm{ICM}}$. Now the saturation sets in when the self-gravity of the shell is dominant over most of the compression phase ( $\S$ 4.2.1). This happens when a substantial fraction of the cloud mass has been swept into a shocked shell. Since $f_{\text {shell }}$ is higher at any $P_{\text {ICM }}$ for a smaller mass cloud, therefore the saturation in $f_{\text {shel1 }}$ also occurs at a smaller value of $P_{\text {ICM }}$.

Thus, depending on the mass spectrum of the molecular clouds in a galactic disk, the smaller mass clouds could be more important in the net contribution toward the star formation rate in starbursts. In a weak central overpressure as seen in M33 (see JD92), only the small-mass disk clouds will become compressed, but not the larger mass clouds.

\subsubsection{Dependence on Overpressure, $P_{\mathrm{ICM}} / P_{\mathrm{GMC}}$}

The overpressure due to the ICM, $P_{\mathrm{ICM}} / P_{\mathrm{GMC}}$, is also an independent input parameter in this problem, as shown in $\S$ 4.1.1. In Figure 5, we plot $f_{\text {shell }}$ versus $P_{\mathrm{ICM}} / P_{\mathrm{GMC}}$ for the overpressure values in the range of $\sim 1-125$. This effectively combines the results for clouds of different radii as plotted in Figure 4. The physical explanation for the behavior of this curve is as given above. In this format, the only input parameter is the external overpressure, and hence the results are applicable for general ICM and cloud parameters, not necessarily restricted to the cases considered in Figure 4.

Note that $f_{\text {shell }}$ is $\sim 0.75-0.80$, that is it is a significant quantity even when the overpressure is only approximately a factor of few. This is because the initial inward shock velocity is proportional to the square root of the ICM pressure (see eq. [1]); hence, even for a small overpressure of a factor of few, a shock is driven in. At later stages of

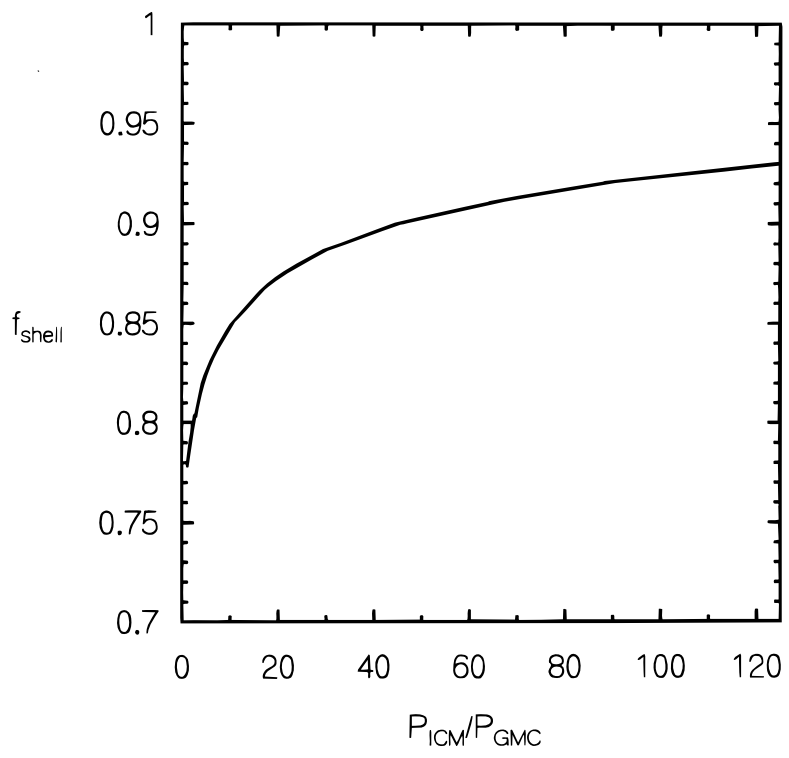

FIG. 5.- A plot of $f_{\text {shell }}$, the fraction of cloud mass compressed at the point of onset of shell instabilities, as a function of the ICM overpressure, $P_{\mathrm{ICM}} / P_{\mathrm{GMC}}$. Here $f_{\text {shell }}$ increases slowly with the increasing overpressure at first and saturates for higher values of the overpressure. Values of $f_{\text {shell }}$ are high even for small factors of overpressure; hence, the triggering of starbursts by overpressure as proposed by JD92 is a general mechanism. compression, when sufficient mass has been swept into the shocked shell, the self-gravity of the shell is the dominant process that decides the inward shock velocity. Thus, even a small overpressure is sufficient to start the process of shock compression, which results eventually in the compression of a substantial fraction of the cloud mass. The resulting $f_{\text {shell }}$ is not too sensitively dependent on the value of the overpressure, especially once the saturation sets in at high overpressure values.

This is an important result because it shows that the scenario for central starbursts triggered in the outer shells of disk clouds via compression by the central overpresssure, as proposed by JD92, is a general mechanism and will operate as long as the central ICM pressure is even a few times higher than the internal effective pressure of an incoming disk GMC

In the case of weak shocks associated with a small value of overpressure, the actual time taken for the onset of instabilities that corresponds to the crossover point would be higher. However, since we are considering self-gravitating clouds to begin with, this does not pose a serious limitation to the formation of instabilities.

In the other extreme limit of high $P_{\mathrm{ICM}} \sim\left(10^{7}-10^{8}\right) k_{\mathrm{B}} \mathrm{K}$ $\mathrm{cm}^{-3}$ as applicable for the starburst galaxies such as NGC 1808 , the value of $f_{\text {shell }}$ is only somewhat larger, $\sim 0.95$ (see $\S 4.3$ ). Finally, it is interesting to note that even in the presence of an ever present pressure reservoir, the fraction of the cloud that becomes compressed is typically $<1$, as was already noted by JD92.

\subsubsection{Mass of Shell Fragments}

Define $M_{\text {inst }}$ to be the mass of the fastest growing instability formed in the shocked shell when the shell becomes unstable:

$$
M_{\text {inst }}=\Sigma_{0} \lambda^{2}=f_{\text {shell }} M_{c} \pi\left(\lambda / l_{\text {shell }}\right)^{2},
$$

where $\Sigma_{0}$ is the surface density of the shell, $\lambda$ is the most unstable wavelength in the shocked shell, and $l_{\text {shell }}=2 \pi r$ is the perimeter of the unshocked cloud at the onset of instability; see $\S 3 . M_{\text {inst }}$ characterizes the mass of a shell fragment and gives an upper limit on the mass of a star cluster formed in the shocked shell. In Figure 6, we plot the ratio $M_{\text {inst }} / M_{c}$; that is, the mass of the instability as a fraction of the cloud mass versus $P_{\mathrm{ICM}}$ for clouds of radii $R_{c}=10 \mathrm{pc}, 20$ pc, and $40 \mathrm{pc}$. The corresponding masses of clouds are $4.0 \times 10^{4} M_{\odot}, 3.2 \times 10^{5} M_{\odot}$, and $2.6 \times 10^{6} M_{\odot}$, respectively $(\S 2.1)$.

For a cloud of given radius, this ratio shows a sharp decrease as $P_{\mathrm{ICM}}$ is increased and its value saturates for large $P_{\text {ICM }}$ values. This can be explained as follows. For a given $\rho_{R}$ and a cloud radius $R_{c}, f_{\text {shell }}$ increases with increasing $P_{\mathrm{ICM}}$ (Fig. 4). On the other hand, $\left(\lambda / l_{\text {shell }}\right)^{2}$ is proportional to $\Sigma_{0}^{-2}$ and hence decreases sharply. Therefore, the net $M_{\text {inst }}$ or $M_{\text {inst }} / M_{c}$ decreases with increasing $P_{\text {ICM }}$. Physically, this means that the number of instabilities in the shell increases with $P_{\text {ICM }}$, and hence $M_{\text {inst }}$, the mass of individual shell fragment, decreases. Since $\lambda / l_{\text {shell }}$ is a sizable fraction $\geq 0.3$ (and not $\ll 1$; see Fig. 3 ), and $f_{\text {shell }}$ is a significant fraction, $\sim 0.8$ (Fig. 4$), M_{\text {inst }}$ is a sizable fraction $(\sim 0.3-0.5)$ of the cloud mass. Thus, $M_{\text {inst }}$ or the upper limit on the mass of the star clusters formed covers a range of values $\sim 10^{4}-10^{6} M_{\odot}$.

At a given $P_{\mathrm{ICM}}$, the ratio $M_{\mathrm{inst}} / M_{c}$ is higher for a larger mass cloud. That is, the fractional and hence the absolute mass of the instability formed is higher for a larger mass 


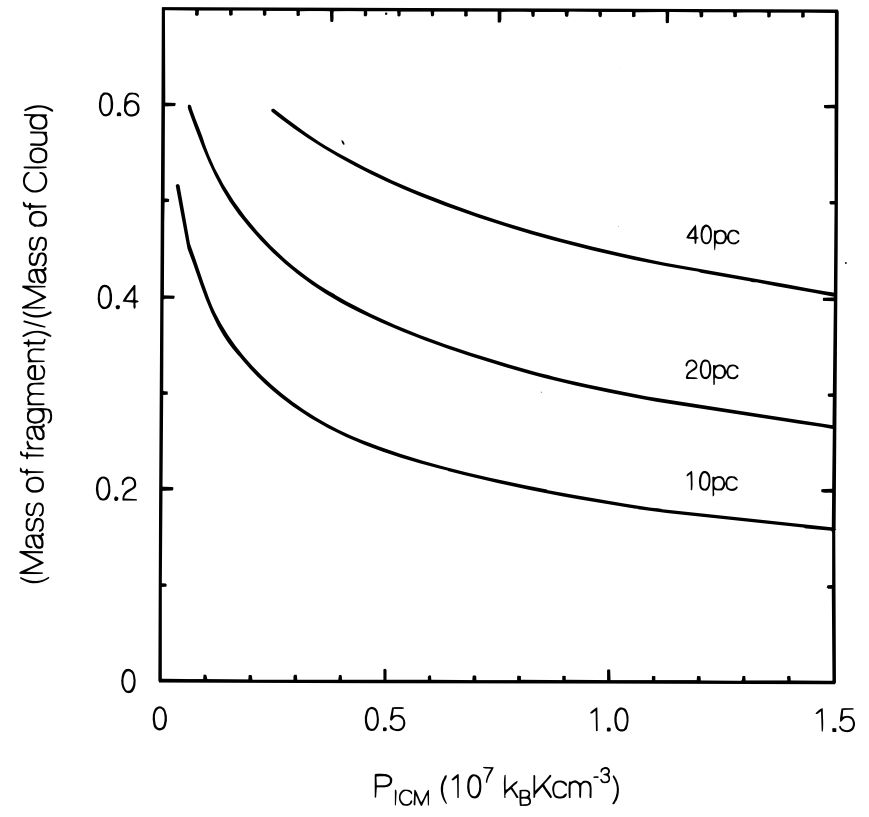

FIG. 6.-A plot of $M_{\text {inst }} / M_{c}$, the mass of an instability or a fragment in the outer shocked shell over the cloud mass, as a function of the ICM pressure, $P_{\mathrm{ICM}}$, for clouds of radii $10 \mathrm{pc}, 20 \mathrm{pc}$, and $40 \mathrm{pc}$. This ratio gives an upper limit on the mass of stellar clusters formed in the shells. $M_{\text {inst }} / M_{c}$ decreases with increasing $P_{\mathrm{ICM}}$. The fractional as well as the absolute mass of the clusters formed is larger for the larger mass clouds.

cloud. From equation (12), it can be shown that $M_{\text {inst }} / M_{c}$ is proportional to $\left(C_{c} / R_{c}\right)^{4}\left(r / R_{c}\right)^{2} /\left(1-r^{2} / R_{c}^{2}\right)$, where $C_{c}$ is the sound speed in the cloud and $r$ is the crossover radius. Now, the value of $r / R_{c}$ is larger for more massive clouds (Fig. 4), and also $C_{c}$ is shown to be linearly proportional to cloud radius for the clouds considered (§ 2.1). Thus, at a given value of $P_{\mathrm{ICM}}$, the ratio $M_{\text {inst }} / M_{c}$ is larger for a more massive cloud.

Note also from Figure 6 that the mass of the instability saturates at high values of $P_{\mathrm{ICM}}$. This can be explained due to the saturation in $f_{\text {shell }}$ occurring at high $P_{\text {ICM }}$, as seen in Figure 4. Figure 6 also shows that the saturation value is smaller for the lower mass clouds. This is due to the smaller saturation value of $f_{\text {shell }}$ (see Fig. 4) and the smaller values of $\lambda / l_{\text {shell }}$ (see $\S 4$ 4.1.1) for the lower mass clouds.

Future high-resolution millimeter-wave interferometric observations with a resolution of approximately a few arcseconds (e.g., Sargent \& Welch 1993) would be able to reveal these few-parsec-size central star clusters in the nearby starburst galaxies.

\subsection{Application to External Galaxies}

In this subsection we apply the above mechanism to real external galaxies for which data are available on the central ICM. These are by necessity nearby galaxies; for these, the spatial resolution is adequate to study the detailed properties of the central gas, as, for example, for IC 342 (Downes et al. 1992) and NGC 1808 (Aalto et al. 1994). The mechanism was applied earlier to the Galaxy and M33 (see JD92).

$$
\text { 4.3.1. IC } 342
$$

IC 342 contains a high concentration of molecular gas $\left(\sim 10^{9} M_{\odot}\right)$ in the central region. The far-infrared and $2 \mu \mathrm{m}$ emission from the center is similar to the central properties of our Galaxy (Eckart et al. 1990). There is a small central bar that is not visible in the optical images (Ishizuki et al. 1990). The recent CO and HCN mapping of the central region of IC 342 by Downes et al. (1992) indicates that the central gas consists of a two-component molecular medium. The low-density ICM has a density of $\sim 150-300 \mathrm{~cm}^{-3}$ and a velocity line width of $\Delta V \sim 50 \mathrm{~km} \mathrm{~s}^{-1}$. The high-density cores are traced mainly by $\mathrm{HCN}$ emission lines and have a density of $\sim 10^{4} \mathrm{~cm}^{-3}$. Therefore, the one-dimensional velocity dispersion of the ICM is given by (see Spitzer 1978)

$$
C_{\mathrm{ICM}}=\Delta V /\left[2(2 \ln 2)^{1 / 2}\right]
$$

Thus, the sound speed in the ICM is $C_{\mathrm{ICM}} \sim 21.2 \mathrm{~km} \mathrm{~s}^{-1}$. The intercloud medium has an average observed density $n_{\text {ICM }} \sim 225 \mathrm{~cm}^{-3}$. Thus, the ICM pressure is equal to $P_{\mathrm{ICM}}=3.4 \times 10^{7} k_{\mathrm{B}} \mathrm{K} \mathrm{cm}^{-3}$, which is 7 times larger than the ICM pressure in the Galaxy. The outer number density of a disk molecular cloud in this galaxy is taken to be similar to our Galaxy, i.e., $n_{\mathrm{GMC}} \sim 100 \mathrm{~cm}^{-3}$ (§ 2.1). This is valid assuming that the cloud sizes and the interstellar UV flux in the two galaxies are comparable (Solomon 1978). The clouds are taken to have radii of $10 \mathrm{pc}, 20 \mathrm{pc}$, and $40 \mathrm{pc}$ as before. Hence, the cloud internal pressure would be as in the disk clouds in the Galaxy. Figure 7 shows how $t_{c}$ and $t_{g}$ change with the evolution of the shock into the cloud for a typical cloud of radius $R_{c}=20 \mathrm{pc}$. At the crossover point, $f_{\text {shell }}=0.91$ in this case. This is larger than the compression of $f_{\text {shell }}=0.85$ resulting for parameters as in the Galaxy; see Figure 2 . For clouds of radii $R_{c}=10 \mathrm{pc}$ and $40 \mathrm{pc}$, the resulting values of $f_{\text {shell }}$ at the respective crossover points are 0.95 and 0.87 , respectively.

The subsequent fragmentation and star formation in the shocked outer shell should lead to starburst in the clouds in the central region of the galaxy following the formalism as in JD92, assuming that the gas infall rate is high enough. The central $1 \mathrm{kpc}$ region of IC 342, however, does not exhibit very high star formation rate and has only a moderate infrared luminosity of $4 \times 10^{9} L_{\odot}$ (Eckart et al. 1990). Therefore, we conclude that the gas infall rate may be small

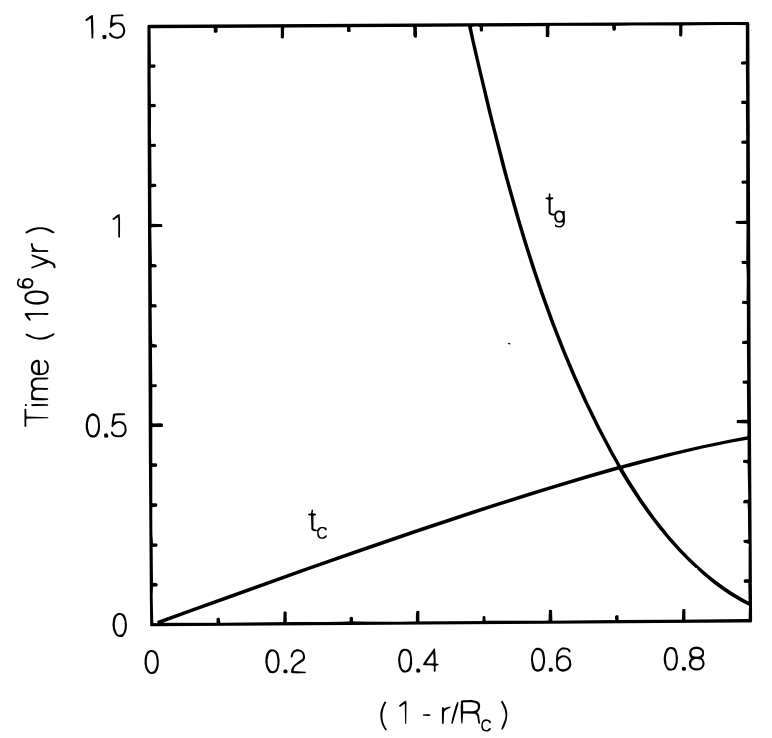

Fig. 7.-A plot of $t_{c}$, the crossing time, and $t_{g}$, the growth time of the fastest growing instability in the shocked shell, vs. $\left(1-r / R_{c}\right)$, the fractional cloud penetration depth for parameters as in IC 342 , so that $P_{\mathrm{ICM}}=3.4$ $\times 10^{7} \mathrm{k}_{\mathrm{B}} \mathrm{K} \mathrm{cm}^{-3}$. Note that the curves cross over at a deeper point; hence, $f_{\text {shell }}$ is higher, equal to 0.91 , than for the case in Fig. 2. 
in this galaxy, perhaps due to the central bar being weak, and that is why no significant star formation is seen in the central regions.

\subsubsection{NGC 1808}

NGC 1808 has nuclear hot spots, strong thermal dust emission, and optical emission lines characteristic of a starburst galaxy (e.g., Aalto et al. 1994). The galaxy may be interacting distantly with NGC 1792 (Dahlem 1992). There also appears to be a bar embedded within the dust lane (Saikia et al. 1990). NGC 1808 was mapped in CO and HCN lines by Aalto et al. (1994). They conclude that the central region has a two-component molecular medium, of which one is a diffuse molecular medium of a high mean density $\sim 2000 \mathrm{~cm}^{-3}$ and line width $\Delta V \sim 30 \mathrm{~km} \mathrm{~s}^{-1}$. The sound velocity in the ICM (from eq. [13]) is thus $C_{\mathrm{ICM}} \sim$ $12.7 \mathrm{~km} \mathrm{~s}^{-1}$. Thus, the ICM pressure $P_{\mathrm{ICM}}$ is very high, equal to $1.1 \times 10^{8} k_{\mathrm{B}} \mathrm{K} \mathrm{cm}^{-3}$. This is 20 times larger than the ICM pressure in the Galaxy.

We assume as before that disk clouds of outer density $n_{\mathrm{GMC}} \sim 100 \mathrm{~cm}^{-3}$ have tumbled into the central region. Clouds of radii $10 \mathrm{pc}, 20 \mathrm{pc}$, and $40 \mathrm{pc}$ were taken as in the previous section. The fractional mass of the shell $f_{\text {shell }}$ at the point of onset of instability is 0.97 for the cloud of $10 \mathrm{pc}$ radius, 0.94 for the cloud of 20 pc radius, and 0.91 for the cloud of 40 pc radius.

Since the galaxy is interacting distantly with NGC 1792 , there should be considerable gas infall. Hence, we expect to see a central starburst in NGC 1808. This agrees with observations because NGC 1808 has a high observed infrared luminosity of $3 \times 10^{10} L_{\odot}$ (Aalto et al. 1994).

\section{DISCUSSION}

We discuss briefly a few general points about the mechanism, as well as the limitations in the analysis presented here.

1. Figure 5 shows that even an overpressure of a factor of, say, 1.5 gives rise to a fairly large value $\sim 0.7$ for the $f_{\text {shell }}$. However, for such small values of overpressure and hence small shock velocities, the shock may not be purely isothermal as assumed here, and hence the assumption of thin shell may not be valid, especially in the early stages of cloud compression. The assumption of thin shell as done here for mathematical convenience would, in this case, result in a slight overestimate of $f_{\text {shell }}$.

2 . In the above analysis, the graininess of the ICM component has been ignored. This is probably acceptable for the larger clouds, but for the smaller clouds of radii $10 \mathrm{pc}$ each, this assumption is not strictly valid. The observational upper limit set on the ICM "cloud" size is a few parsecs (Bally et al. 1987, 1988). This would result in one-sided compression or a ballistic motion of the disk cloud on impact with the ICM constituents rather than resulting in a uniform compression, as assumed here.

3. In $\S 4.3$, the shock compression has been calculated for IC 342 and for NGC 1808. In both cases, since the ICM density $n_{\mathrm{ICM}}$ is higher than $n_{c}$, the shock velocity $V_{s}$ is larger than $C_{\mathrm{ICM}}$, the sound speed in the ICM. This means that the ICM pressure reservoir does not remain in contact with the inward moving postshock shell of gas, and hence, strictly speaking, our treatment of cloud compression will not hold. In this case, the cloud compression may occur in a quasistatic fashion. By ignoring this complicated process, we have probably overestimated slightly the value of $f_{\text {shell }}$ for galaxies with large $n_{\mathrm{ICM}}$ values. However, in both these cases, since the initial $V_{s}$ set by the ICM pressure is very high, the self-gravity of the shell rises rapidly and after a certain stage it will be the dominant accelerating mechanism that would drive the shock inward. Hence, interestingly, our treatment of shock compression and the resulting value of $f_{\text {shell }}$ may not be a bad approximation of cloud compression even when the shock velocity exceeds the ICM sound speed.

4. The starburst mechanism proposed in this paper will break down when the infall rate of disk GMCs becomes very large because, in this case, the pressure reservoir cannot be replenished at the rate required. Such a situation is expected to arise during the late stages of mergers of gasrich spiral galaxies such as Arp 220, in which the infall time is reduced considerably to $\sim 10^{7}$ yr (Norman 1992; JD92), and the resulting gas infall rates can be high, $\geq 100 M_{\odot}$ $\mathrm{yr}^{-1}$. In this case, alternative triggering mechanisms for star formation such as cloud-cloud collisions (e.g., Scoville, Sanders, \& Clemens 1986) would probably be important.

5. We do not consider the value of $n_{c}$, the outer number density in a cloud, to be less than $100 \mathrm{~cm}^{-3}$ (§ 2.1). A lower value of $n_{c}$ is ruled out from the UV shelf-shielding argument unless the cloud has a large, low-density halo or envelope of atomic hydrogen. Such envelopes have been detected for some molecular clouds (Deiter 1960; Wannier, Lichten, \& Morris 1983; Wannier et al. 1993). If this envelope remains intact as the cloud falls to the central region, then the details of shock propagation will be different. The value of $f_{\text {shell }}$ in that case would probably be smaller than is estimated here for clouds without an $\mathrm{H} \mathrm{I}$ envelope.

6. While this work is done with the direct application for starburst galaxies in mind, the above results for shock compression by overpressure are general, and the resulting star formation in outer shells is a general mechanism. Hence, the results could be applied for other astronomical situations, say, for example, for compression of clumps inside molecular clouds by overpresssure due to stellar winds (Boss 1995), or for the compression of a cometary globule.

\section{SUMMARY}

The dependence of the shock compression of a disk cloud due to a central overpressure (JD92) on various parameters has been studied. We find that the main physical input parameters in the problem are the external (ICM) pressure, the cloud density, and the cloud radius. Alternatively, the independent input parameter is the overpressure on the cloud, $P_{\mathrm{ICM}} / P_{\mathrm{GMC}}$. The clouds are taken to be nearly virialized with a $1 / r$ internal density profile as observed (Sanders et al. 1985), and are all assumed to have the same outer number density.

We have spanned exhaustively the parameter space for the disk GMC and the central ICM properties covering the overpressure range of $\sim 1-125$, and for clouds of radii $10 \mathrm{pc}$, $20 \mathrm{pc}$, and $40 \mathrm{pc}$. We find that for this wide range of input parameters, the fraction of cloud mass compressed at the onset of instabilities, $f_{\text {shell }}$, is high $\sim 0.75-0.90$, and it is fairly insensitive to the actual value of the ICM pressure. This is because the initial shock velocity is proportional to the square root of the external (ICM) pressure; and at later stages it is set mainly by the self-gravity of the shocked shell. Thus, the triggering of starbursts by overpressure as pro- 
posed by JD92 is a general mechanism and will operate as long as the ICM pressure is even a factor of few higher than the effective internal pressure of an incoming disk GMC.

For a given ICM pressure, a greater fraction of a smaller mass cloud is compressed than is the case for a large mass cloud. In a region of weak overpressure as in the central region of M33, only the low-mass disk clouds would undergo compression and could be the sites of the consequent starburst.

The resulting mass of an instability in the shell of a cloud sets an upper limit to the mass of the star cluster formed. This is a significant fraction, $\sim 0.3-0.5$ of the cloud mass, and it covers values $\sim 10^{4}-10^{6} M_{\odot}$ and decreases with increasing ICM pressure. The fractional as well as the absolute mass of a star cluster formed is higher for the larger mass clouds. Future high-resolution millimeter-wave observations with the resolution of approximately a few arcseconds (e.g., Sargent \& Welch 1993) would be able to detect these few-parsec-size central star clusters in the nearby starburst galaxies.

Since the observed ICM parameters for IC 342 and NGC 1808 are known, we have applied the mechanism to these galaxies. We find that in both cases a large mass fraction $(>0.9)$ of an incoming disk cloud will be compressed by the central overpressure. However, the ultimate central luminosity from star formation will depend on the gas infall rate in these galaxies. Hence, NGC 1808 with gas infall due to galaxy interaction shows a central starburst with an infrared luminosity of $3 \times 10^{10} L_{\odot}$, while IC 342 , with probably a lower gas infall rate, does not show a central starburst.

C. J. would like to thank Phil Solomon, Nick Scoville, George Helou, and Alan Boss for useful discussions. M. D. would like to thank CSIR, India, for a junior research fellowship. We thank the referee for the comments on the paper.

\section{REFERENCES}

Aalto, S., Booth, R. S., Black, J. H., Koribalski, B., \& Wielebinski, R. 1994, A\&A, 286, 365

Bally, J., Stark, A. A., Wilson, R. W., \& Henkel, C. 1987, ApJS, 65, 13

1988, ApJ, 324, 223

Boss, A. 1995, ApJ, 439, 224

Dahlem, M. 1992, A\&A, 264, 483

Deiter, N. H. 1960, ApJ, 132, 49

Downes, D., Radford, S. J. E., Guilloteau, S., Guelin, M., Greve, A., \& Morris, D. 1992, A\&A, 262, 424

Eckart, A., Downes, D., Genzel, R., Harris, A. I., Jaffe, D. T., \& Wild, W. 1990, ApJ 348,433

Güsten, R. 1989, in IAU Symp. 136, The Center of the Galaxy, ed. M. Morris (Dordrecht: Kluwer), 89

Ishizuki, S., Kawabe, R., Ishiguro, M., Kumura, S. O., \& Norita, K. I. 1990 Nature, 344, 224

Jog, C. J., \& Das, M. 1992, ApJ, 400, 476 (JD92)

Jog, C. J., \& Solomon, P. M. 1984, ApJ, 276, 114

Jog, C. J., \& Solomon, P. M.

Norman, C. A. 1992, in Massive Stars in Starbursts, ed. C. Leitherer, T. M. Heckman, C. A. Norman, \& N. R. Walborn (Cambridge: Cambridge Univ. Press), 271

Saikia, D. J., Unger, S. W., Pedlar, A., Yates, G. J., Axon, D. J. Wolstencraft, R. D., Taylor, K., \& Gyldenkerne, K. 1990, MNRAS, 245, 397
Sanders, D. B., Scoville, N. Z., \& Solomon, P. M. 1985, ApJ, 289, 373

Sanders, R. H. 1989, in IAU Symp. 136, The Center of the Galaxy, ed. M. Morris (Dordrecht: Kluwer), 77

Sargent, A. I., \& Welch, J. E. 1993, ARA\&A, 31, 297

Scoville, N. Z., \& Sanders, D. B. 1987, in Interstellar Processes, ed. D. J. Hollenbach \& H. A. Thronson, Jr. (Dordrecht: Reidel), 21

Scoville, N. Z., Sanders, D. B., \& Clemens, D. P. 1986, ApJ, 310, L47

Solomon, P. M. 1978, in Infrared Astronomy, ed. G. Setti \& G. G. Fazio (Dordrecht: Reidel), 97

Spitzer, L. 1978, The Physical Processes in the Interstellar Medium (New York: John Wiley)

Stark, A. A., Bally, J., Wilson, R. W., \& Pound, M. W. 1989, in IAU Symp. 136, The Center of the Galaxy, ed. M. Morris (Dordrecht: Kluwer), 129 Toomre, A. 1964, ApJ, 139, 1217

Wannier, P. G., Andersson, B.-G., Federman, S. R., Lewis, B. M., Viala, Y. P., \& Shaya, E. 1993, ApJ, 407, 163

Wannier, P. G.. Lichten, S. M. \& Morris, M. 1983 ApJ, 268, 727

Wilson, C. D., \& Scoville, N. Z. 1990, ApJ, 363, 435

Young, J. S. 1993, in The Environment and Evolution of Galaxies, ed. J. M. Shull \& H. A. Thronson (Dordrecht: Kluwer), 295 